\title{
Taxonomic Study of Lancefield Streptococcal Groups C, G, and L (Streptococcus dysgalactiae) and Proposal of $S$. dysgalactiae subsp. equisimilis subsp. nov.
}

\author{
P. VANDAMME, ${ }^{1,2 *}$ B. POT, ${ }^{3}$ E. FALSEN,${ }^{4}$ K. KERSTERS,${ }^{1}$ AND L. A. DEVRIESE ${ }^{5}$ \\ Laboratory of Microbiology ${ }^{1}$ and BCCM/LMG Culture Collection, ${ }^{3}$ Faculty of Sciences, and Laboratory of \\ Microbiology, Faculty of Veterinary Medicine, ${ }^{5}$ University of Ghent, Ghent, and Laboratory of Medical \\ Microbiology, University Hospital Antwerp ULA, Antwerp, ${ }^{2}$ Belgium, and Department of \\ Clinical Bacteriology, University of Göteborg, Göteborg, Sweden ${ }^{4}$
}

\begin{abstract}
Streptococcus dysgalactiae consists of at least five distinct subgroups on the basis of serogroups, biotypes, and hosts. A chemotaxonomic and phenotypic examination of $80 \mathrm{~S}$. dysgalactiae strains representing the known diversity within this species and 49 reference strains representing all members of the streptococcal pyogenic species group revealed two subpopulations of strains within $S$. dysgalactiae. The name $S$. dysgalactiae subsp. dysgalactiae is proposed for strains of animal origin. These strains belong to Lancefield serogroups $\mathrm{C}$ and $\mathrm{L}$, are alpha-, beta-, or nonhemolytic, and do not exhibit streptokinase activity on human plasminogen or proteolytic activity on human fibrin. The name $S$. dysgalactiae subsp. equisimilis is proposed for human isolates. These strains belong to Lancefield serogroups $\mathrm{C}$ and $\mathrm{G}$, are beta-hemolytic, and exhibit streptokinase activity on human plasminogen and proteolytic activity on human fibrin.
\end{abstract}

In 1936, Frost and Engelbrecht proposed the name Streptococcus equisimilis for a group of beta-hemolytic streptococci belonging to Lancefield serogroup $\mathrm{C}(10,12)$. S. equisimilis strains were isolated from the nose, throat, vagina, and skin of humans and were thought to be uncommon in domestic animals (2). About a decade later, the bovine organism Streptococcus dysgalactiae was reported to be identical to $S$. equisimilis, except for the absence of beta-hemolysis (2). However, in 1980 , both species names lost standing in nomenclature when they were not included on the Approved Lists of Bacterial Names (15). S. dysgalactiae was revived in 1983, but was restricted to the alpha-hemolytic, group C strains of bovine origin (11). Subsequently, Farrow and Collins (9) demonstrated that $S$. dysgalactiae, $S$. equisimilis, and streptococci belonging to serogroups $\mathrm{G}$ (the large-colony-forming strains) and $\mathrm{L}$ all exhibited high levels of DNA-DNA binding and therefore belonged to a single species, $S$. dysgalactiae. Except for Streptococcus agalactiae (serogroup B), the serological groups designed by Lancefield (12) do not correspond to individual species and for species other than $S$. agalactiae are useful primarily for differentiating infraspecific biovars or ecovars (5). In the case of $S$. dysgalactiae, serotyping made species level identification extremely complex. Among the group $\mathrm{C}$ streptococci, the following five taxa have been differentiated: (i) the human, beta-hemolytic strains; (ii) the porcine, beta-hemolytic strains previously named $S$. equisimilis, which belong to $S$. dysgalactiae; (iii) the bovine, alpha-hemolytic strains, which are the $S$. dysgalactiae strains defined by Garvie et al. (11); (iv) the equine, beta-hemolytic strains which belong to the two subspecies of Streptococcus equi (S. equi subsp. equi and S. equi subsp. zooepidemicus); and (v) the human, small-colony-forming, beta-hemolytic strains which belong to the "Streptococcus milleri" group $(3,5)$. Among the group $G$ streptococci, the following three taxa have been differentiated: (i) the human, large-col-

\footnotetext{
* Corresponding author. Mailing address: Laboratorium voor Microbiologie, Ledeganckstraat 35, B-9000 Ghent, Belgium. Phone: (32)9.264.51.14. Fax: (32)9.264.53.46. Electronic mail address: Peter Vandamme@rug.ac.be.
}

ony-forming, beta-hemolytic strains which belong to $S$. dysgalactiae; (ii) the human, small-colony-forming, beta-hemolytic strains which belong to the " $S$. milleri" group; and (iii) the bovine, canine, and feline, beta-hemolytic strains, which belong to Streptococcus canis (6). Finally, as explained above, the beta-hemolytic group $\mathrm{L}$ streptococci also belong to $S$. dysgalactiae (9).

Although invalid, the name $S$. equisimilis is still widely used $(3,7,13)$, and the nomenclature of the entire group remains confused. In order to shed light on the taxonomy of these organisms, we examined $80 S$. dysgalactiae strains representing the various subgroups within this species and 49 additional reference strains by using whole-cell protein electrophoresis, a technique that has been shown to be very useful for differentiating strains at or below the species level (4). The phenotypic characteristics of the clusters of strains obtained were extensively examined, and a new classification for these bacteria is proposed.

\section{MATERIALS AND METHODS}

Bacterial strains. Type and other reference strains were obtained from international culture collections as listed in Table 1 . The other strains examined were mostly our field isolates (isolates of L.A.D.). All of the strains used and their sources are listed in Table 1. Bacteriological purity was checked by plating and examining living and Gram-stained cells.

Whole-cell protein analysis. All strains were grown for $24 \mathrm{~h}$ on brain heart infusion agar (catalog no. 0037-17-8; Difco Laboratories, Detroit, Mich.) incubated at 36 to $37^{\circ} \mathrm{C}$ in a microaerobic atmosphere containing approximately $5 \%$ $\mathrm{O}_{2}, 10 \% \mathrm{CO}_{2}$, and $85 \% \mathrm{~N}_{2}$. Preparation of cellular protein extracts, polyacrylamide gel electrophoresis (PAGE), a densitometric analysis, normalization and interpolation of the protein profiles, and a numerical analysis were performed as described by Pot et al. (14) by using the GelCompar 3.1 software package (Applied Maths, Kortrijk, Belgium). The profiles were recorded and stored on a PC computer. The levels of similarity bctween pairs of traces were expressed by the Pearson product moment correlation coefficient converted for convenience to a percentage.

Physiological tests. Strains were grown on Columbia agar (Lab M, Paisley, United Kingdom) supplemented with $5 \%$ cattle blood at $37^{\circ} \mathrm{C}$ in an atmosphere containing $5 \% \mathrm{CO}_{2}$ in air. Serological grouping with Lancefield group A, B, C, $\mathrm{D}, \mathrm{F}$, and $\mathrm{G}$ antigens was carried out by using an Oxoid streptococcal grouping kit (Unipath, Basingstoke, United Kingdom). Acid $(0.2 \mathrm{~N} \mathrm{HCl})$ Lancefield extracts of strains that did not react in these tests were tested by using group $\mathrm{L}$ antiserum obtained from D. G. Groothuis (National Institute of Public Health and Environmental Protection, Bilthoven, The Netherlands).

Streptokinase activity was determined on fibrin-plasma plates made up from 
TABLE 1. Strains used

\begin{tabular}{|c|c|c|c|c|}
\hline Name & LMG no. ${ }^{a}$ & Other designation $(\mathrm{s})^{a}$ & Depositor ${ }^{a, h}$ & Source and/or group \\
\hline S. dysgalactiae subsp. dysgalactiae & 14602 & Ton 516 & & Bovine, group C \\
\hline S. dysgalactiae subsp. dysgalactiae & 14604 & Ton 517 & & Bovine, group C \\
\hline S. dysgalactiae subsp. dysgalactiae & 14605 & Ton 528 & & Bovine, group C \\
\hline S. dysgalactiae subsp. dysgalactiae & 14606 & Higgins 79 & Higgins & Chicken, group L \\
\hline S. dysgalactiae subsp. dysgalactiae & 15732 & STR 656 & & Porcine, group C \\
\hline S. dysgalactiae subsp. dysgalactiae & 15733 & STR 655, CCUG 27659 & & Porcine, group C \\
\hline S. dysgalactiae subsp. dysgalactiae & 15734 & STR 654, CCUG 27658 & & Porcine, group C \\
\hline S. dysgalactiae subsp. dysgalactiae & 15735 & STR 653, CCUG 27657 & & Porcine, group $\mathrm{C}$ \\
\hline S. dysgalactiae subsp. dysgalactiae & 15736 & T3583 & Hommez & Porcine, group C \\
\hline S. dysgalactiae subsp. dysgalactiae & 15737 & T3588 & Hommez & Porcine, group C \\
\hline S. dysgalactiae subsp. dysgalactiae & 15743 & $94 / 1479$ & & Iguana, group C \\
\hline S. dysgalactiae subsp. dysgalactiae & 15744 & Ton 332 & & Porcine, group C \\
\hline S. dysgalactiae subsp. dysgalactiae & 15748 & STR 240 & & Bovine, group $\mathrm{C}$ \\
\hline S. dysgalactiae subsp. dysgalactiae & 15749 & STR 490 & & Bovine, group C \\
\hline S. dysgalactiae subsp. dysgalactiae & 15750 & STR 273 & & Bovine, group C \\
\hline S. dysgalactiae subsp. dysgalactiae & 15751 & STR T64 & Hommez & Bovine, group L \\
\hline S. dysgalactiae subsp. dysgalactiae & 15752 & STR T12 & Hommez & Bovine, group L \\
\hline S. dysgalactiae subsp. dysgalactiae & 15753 & STR 449, CCUG 27666 & & Bovine, group L \\
\hline S. dysgalactiae subsp. dysgalactiae & 15754 & Higgins 58 & Higgins & Chicken, group $\mathrm{L}$ \\
\hline S. dysgalactiae subsp. dysgalactiae & 15755 & Higgins 67 & Higgins & Chicken, group L \\
\hline S. dysgalactiae subsp. dysgalactiae & 15756 & STR 772 & & Porcine, group L \\
\hline S. dysgalactiae subsp. dysgalactiae & 15757 & STR 771 & & Porcine, group L \\
\hline S. dysgalactiae subsp. dysgalactiae & 15758 & Ton 130 & & Canine, group $\mathrm{C}$ \\
\hline S. dysgalactiae subsp. dysgalactiae & 15759 & Ton 133 & & Canine, group C \\
\hline S. dysgalactiae subsp. dysgalactiae & 15766 & Ma R & & Bovine, group C \\
\hline S. dysgalactiae subsp. dysgalactiae & 15824 & CCUG 28112 & CCUG & Equine, group $\mathrm{C}$ \\
\hline S. dysgalactiae subsp. dysgalactiae & 15830 & CCUG 11671A & CCUG & Canine, group L \\
\hline S. dysgalactiae subsp. dysgalactiae & 15831 & CCUG 27665 & CCUG & Canine, group $\mathbf{L}$ \\
\hline S. dysgalactiae subsp. dysgalactiae & 15832 & CCUG 27666 & CCUG & Bovine, group L \\
\hline S. dysgalactiae subsp. dysgalactiae & 15833 & CCUG 28114 & CCUG & Equine uterus, group C \\
\hline S. dysgalactiae subsp. dysgalactiae & 15834 & CCUG 28115 & CCUG & Equine blood, group C \\
\hline S. dysgalactiae subsp. dysgalactiae & 15835 & CCUG 28116 & CCUG & Canine vagina, group $\mathrm{C}$ \\
\hline S. dysgalactiae subsp. dysgalactiae & 15836 & CCUG 28120 & CCUG & Porcine joint, group $\mathrm{C}$ \\
\hline S. dysgalactiae subsp. dysgalactiae & 15896 & STR 785 & & Canine, group C \\
\hline S. dysgalactiae subsp. dysgalactiae & 15897 & STR 782 & & Canine, group $\mathrm{C}$ \\
\hline S. dysgalactiae subsp. dysgalactiae & 15899 & CCUG 28113 & CCUG & Porcine abscess, group C \\
\hline S. dysgalactiae subsp. dysgalactiae & 15900 & CCUG 28117 & CCUG & Porcine, group C \\
\hline S. dysgalactiae subsp. dysgalactiae & 15901 & CCUG 28118 & CCUG & Equine throat, group C \\
\hline S. dysgalactiae subsp. dysgalactiae & 15902 & CCUG 28119 & CCUG & Equine abscess, group $\mathrm{C}$ \\
\hline S. dysgalactiae subsp. dysgalactiae & 15931 & STR 284 & & Canine, group $\mathrm{C}$ \\
\hline S. dysgalactiae subsp. dysgalactiae & $16023^{\mathrm{T}}$ & NCFB $2023^{\mathrm{T}}$, LMG $15885^{\mathrm{T}}$ & NCFB & Bovine, group C \\
\hline S. dysgalactiae subsp. dysgalactiae & 16024 & NCFB 2024 & NCFB & Bovine mastitis, group $\mathrm{C}$ \\
\hline S. dysgalactiae subsp. dysgalactiae & 16025 & NCFB 2043 & NCFB & Bovine milk, group C \\
\hline S. dysgalactiae subsp. dysgalactiae & 16027 & NCFB 2648 & NCFB & Animal, group L \\
\hline S. dysgalactiae subsp. dysgalactiae & 16029 & NCFB 2652 & NCFB & Canine, group L \\
\hline S. dysgalactiae subsp. equisimilis & 15738 & API 79.12.012 & API & Human, group C \\
\hline S. dysgalactiae subsp. equisimilis & 15739 & API 81.10 .028 & API & Human, group C \\
\hline S. dysgalactiae subsp. equisimilis & 15740 & API 84.11 .160 & API & Human, group C \\
\hline S. dysgalactiae subsp. equisimilis & 15745 & API 83.02 .040 & API & Human, group $\mathrm{G}$ \\
\hline S. dysgalactiae subsp. equisimilis & 15746 & API 79.01 .052 & API & Human, group $\mathrm{G}$ \\
\hline S. dysgalactiae subsp. equisimilis & 15747 & API 86.04 .100 & API & Human, group $\mathrm{G}$ \\
\hline S. dysgalactiae subsp. equisimilis & 15818 & CCUG 1845 & CCUG & Human, group C \\
\hline S. dysgalactiae subsp. equisimilis & 15819 & CCUG 4211 & CCUG & Human vagina, group $\mathrm{C}$ \\
\hline S. dysgalactiae subsp. equisimilis & 15820 & CCUG 18742 & CCUG & Human, group C \\
\hline S. dysgalactiae subsp. equisimilis & 15821 & CCUG 20596 & CCUG & Human blood, group C \\
\hline S. dysgalactiae subsp. equisimilis & 15822 & CCUG 27621 & CCUG & Human, group C \\
\hline S. dysgalactiae subsp. equisimilis & 15823 & CCUG 27622 & CCUG & Human, group C \\
\hline S. dysgalactiae subsp. equisimilis & 15825 & CCUG 32016 & CCUG & Human, group C \\
\hline S. dysgalactiae subsp. equisimilis & 15826 & CCUG 32017 & CCUG & Human, group C \\
\hline S. dysgalactiae subsp. equisimilis & 15828 & CCUG 32019 & CCUG & Human, group C \\
\hline S. dysgalactiae subsp. equisimilis & 15829 & CCUG 27483 & CCUG & Human, group $\mathrm{G}$ \\
\hline S. dysgalactiae subsp. equisimilis & 15837 & CCUG 32201 & CCUG & Human blood, group C \\
\hline S. dysgalactiae subsp. equisimilis & 15839 & CCUG 33802 & CCUG & Human, group G \\
\hline S. dysgalactiae subsp. equisimilis & 15840 & CCUG 1483 & CCUG & Human, group C \\
\hline S. dysgalactiae subsp. equisimilis & 15841 & CCUG 6713 & CCUG & Human, group C \\
\hline S. dysgalactiae subsp. equisimilis & 15842 & CCUG 21557 & CCUG & Human, group C \\
\hline S. dysgalactiae subsp. equisimilis & 15843 & CCUG 27478 & CCUG & Human, group C \\
\hline S. dysgalactiae subsp. equisimilis & 15844 & CCUG 27479 & CCUG & Human, group C \\
\hline S. dysgalactiae subsp. equisimilis & 15845 & CCUG 27480 & CCUG & Human, group $\mathrm{C}$ \\
\hline
\end{tabular}


TABLE 1-Continued

\begin{tabular}{|c|c|c|c|c|}
\hline Name & LMG no. ${ }^{a}$ & Other designation $(\mathrm{s})^{a}$ & Depositor ${ }^{a, b}$ & Source and/or group \\
\hline S. dysgalactiae subsp. equisimilis & 15846 & CCUG 502 & CCUG & Human, group $\mathrm{G}$ \\
\hline S. dysgalactiae subsp. equisimilis & 15847 & CCUG 1859 & CCUG & Human, group $\mathrm{G}$ \\
\hline S. dysgalactiae subsp. equisimilis & 15848 & CCUG 7975 & CCUG & Human, group G \\
\hline S. dysgalactiae subsp. equisimilis & 15849 & CCUG 15679 & CCUG & Human, group $\mathrm{G}$ \\
\hline S. dysgalactiae subsp. equisimilis & 15850 & CCUG 15680 & CCUG & Human, group G \\
\hline S. dysgalactiae subsp. equisimilis & 15851 & CCUG 24070 & CCUG & Human throat, group $\mathrm{G}$ \\
\hline S. dysgalactiae subsp. equisimilis & 15852 & CCUG 26147 & CCUG & Human wound, group G \\
\hline S. dysgalactiae subsp. equisimilis & 15853 & CCUG 27477 & CCUG & Human, group G \\
\hline S. dysgalactiae subsp. equisimilis & 15854 & CCUG 27482 & CCUG & Human, group G \\
\hline S. dysgalactiae subsp. equisimilis & $16026^{\mathrm{T}}$ & NCFB $1356^{\mathrm{T}}$ & NCFB & Human, group G \\
\hline S. dysgalactiae subsp. equisimilis & 15903 & CCUG 28238 & CCUG & Human throat, group $\mathrm{C}$ \\
\hline S. agalactiae & $14694^{\mathrm{T}}$ & CCUG $4208^{\mathrm{T}}$ & CCUG & Bovine milk \\
\hline S. agalactiae & 14747 & STR 765 & & Bovine mastitis \\
\hline S. agalactiae & 14847 & SHV 357 & Hommez & Porcine \\
\hline S. agalactiae & 15084 & 18RS21 & Melin & Human \\
\hline S. agalactiae & 15085 & M732 & Melin & Human \\
\hline S. agalactiae & 15095 & 455889 & Melin & Human, respiratory infection \\
\hline S. anginosus & $14502^{\mathrm{T}}$ & CCUG $27298^{\mathrm{T}}$ & CCUG & \\
\hline S. anginosus & 14612 & STR 778 & & Canine \\
\hline S. anginosus & 14613 & STR 200 & & Canine \\
\hline S. anginosus & 14696 & CCUG 223 & CCUG & \\
\hline S. anginosus & 15994 & CCUG 28467 & CCUG & Human \\
\hline S. canis & 14833 & CCUG 27660, STR 290 & & Canine \\
\hline S. canis & 14834 & STR 642 & & Feline \\
\hline S. canis & $15890^{\mathbf{T}}$ & STR-T1 ${ }^{\mathrm{T}}$ & & Bovine mastitis \\
\hline S. canis & 15891 & $21650-1 / 473$ & & Mink \\
\hline 5. canis & 15892 & 03342-I106 & & Fox \\
\hline S. canis & 15893 & $20837 / 7820$ & & Fox \\
\hline S. canis & 15894 & $23668 / 2140$ & & Mink \\
\hline S. equi subsp. zooepidemicus & 15760 & Ton6 & & Equine \\
\hline S. equi subsp. zooepidemicus & 15761 & P11264 & & Equine \\
\hline S. equi subsp. zooepidemicus & 15762 & P13997 & & Equine \\
\hline S. equi subsp. zooepidemicus & 15763 & Ton 174 & & Equine \\
\hline S. equi subsp. equi & 15764 & STR 674 & & Equine \\
\hline S. equi subsp. equi & $15886^{\mathrm{T}}$ & CCUG $23255^{\mathrm{T}}$ & CCUG & Equine \\
\hline S. equi subsp. zooepidemicus & 15888 & CCUG 23257 & CCUG & \\
\hline S. equi subsp. zooepidemicus & 15889 & CCUG 23258 & CCUG & \\
\hline S. equi subsp. zooepidemicus & $16030^{\mathrm{T}}$ & NCFB $1358^{\mathrm{T}}, \mathrm{LMG} 15887^{\mathrm{T}}$ & NCFB & Bovine mastitis \\
\hline S. hyointestinalis & $14579^{\mathrm{T}}$ & $\mathrm{S} 93^{\mathrm{T}}$ & & Porcine \\
\hline S. hyointestinalis & 14581 & S85 & & Porcine \\
\hline S. hyointestinalis & 14582 & EV 28b & & Porcine \\
\hline S. iniae & $14520^{\mathrm{T}}$ & CCUG $27303^{\mathrm{T}}$ & CCUG & Dolphin \\
\hline S. iniae & 14521 & CCUG 27623 & CCUG & Dolphin \\
\hline S. parauberis & 12173 & NCFB 2018, LMG 14377 & NCFB & Bovine mastitis \\
\hline S. parauberis & $12174^{\mathrm{T}}$ & NCFB $2020^{\mathrm{T}}, \mathrm{LMG} 14376^{\mathrm{T}}$ & NCFB & Bovine mastitis \\
\hline S. porcinus & 14615 & STR 827 & & Human \\
\hline S. porcinus & $15980^{\mathrm{T}}$ & CCUG 27628 & CCUG & Porcine \\
\hline S. porcinus & 15981 & CCUG 27629 & CCUG & Porcine \\
\hline S. pyogenes & 14237 & CIP 56.58 & CIP & $\begin{array}{l}\text { Patient with rheumatic fever, } \\
\text { throat }\end{array}$ \\
\hline S. pyogenes & 14238 & CIP 70.3 & CIP & $\begin{array}{l}\text { Patient with bronchopneumonia, } \\
\text { pleural fluid }\end{array}$ \\
\hline S. pyogenes & $14700^{\mathrm{T}}$ & CCUG $4207^{\mathrm{T}}$ & CCUG & \\
\hline S. pyogenes & 15855 & A4260 & Goossens & Human throat \\
\hline S. pyogenes & 15856 & A6825 & Goossens & Human throat \\
\hline S. pyogenes & 15857 & H1667 & Goossens & Human throat \\
\hline S. pyogenes & 15858 & $\mathrm{C} 8185$ & Goossens & Human throat \\
\hline S. pyogenes & 15859 & P4091 & Goossens & Human throat \\
\hline S. shiloi & $15978^{\mathrm{T}}$ & CIP $103769^{\mathrm{T}}$ & CIP & Fish, brain \\
\hline
\end{tabular}


TABLE $1-$ Continued

\begin{tabular}{|c|c|c|c|c|}
\hline Name & LMG no. ${ }^{a}$ & Other designation $(\mathrm{s})^{a}$ & Depositor ${ }^{a, b}$ & Source and/or group \\
\hline S. uberis & 14395 & STR 192 & & Duck, lung \\
\hline S. uberis & 14610 & STR 203 & & Bovine \\
\hline S. uberis & 14686 & ATCC 13386 & ATCC & \\
\hline
\end{tabular}

${ }^{a}$ API, Appareils et Procédés d'Identification, La-Balme-les-Grottes, Montalieu-Vercieu, France; ATCC, American Type Culture Collection, Rockville, Md.; CCUG, Culture Collection of the University of Göteborg, Department of Clinical Bacteriology, University of Göteborg, Göteborg, Sweden; CIP, Collection bactérienne de l'Institut Pasteur, Paris, France; Goossens, H. Goosens, University Hospital Antwerp UIA, Antwerp, Belgium; Higgins, R. Higgins, Department of Pathology and Microbiology, Faculty of Veterinary Medicine, University of Montreal, Sainte-Hyacinthe, Canada; Hommez, J. Hommez, Regional Veterinary Investigation Centre, Torhout, Belgium; LMG, Laboratorium voor Microbiologie Gent Culture Collection, Universiteit Ghent, Gent, Belgium; Melin, P. Melin, Centre Hospitalier Universitaire de Liège, Service de Microbiologie Médicinale, Liège, Belgium; NCFB, National Collection of Food Bacteria, Agricultural and Food Research Council, Institute of Food Research, Reading Laboratory, Reading, Berkshire, United Kingdom.

${ }^{b}$ Our isolate if not indicated otherwise.

Columbia blood agar base supplemented with $1 \mathrm{~g}$ of bovine fibrinogen fraction 1 (catalog no. F-8630; Sigma) per liter and $0.5 \%$ human plasma. Fibrinogen (100 $\mathrm{mg}$ ) was first dissolved in $10 \mathrm{ml}$ of phosphate-buffered saline and warmed for 10 $\mathrm{min}$ in a shaking water bath at $37^{\circ} \mathrm{C}$. This preparation was then mixed with 100 $\mathrm{ml}$ of melted blood agar base and incubated for $10 \mathrm{~min}$ at $56^{\circ} \mathrm{C}$ to allow for precipitation of fibrin. Plates were poured immediately after plasma was added and were stored for a maximum of 3 days. A plate containing the same base and bovine fibrin without plasma was used as a control. In other tests, canine, porcine, equine, bovine, and avian (chicken) plasmas were used as plasminogen sources at the same concentration. A limited number of strains giving positive or negative results on Columbia agar supplemented with bovine fibrin and plasma were also tested on brain heart infusion agar (Oxoid, Basingstoke, United Kingdom) and on nutrient agar (Oxoid) similarly supplemented with human plasma and bovine fibrin. Strains were spot inoculated onto the plates, and clearing of bovine fibrin on plates containing plasma and not on plates containing only fibrin was considered evidence of activation of plasminogen to plasmin (streptokinase) activity. Reactions occurring on plasma-free as well as on plasma-supplemented plates were interpreted as evidence of fibrinolysis caused by protease activity This activity was confirmed by tests on casein plates (Columbia agar supplemented with $0.5 \%$ skim milk) and gelatin plates (Columbia agar supplemented with $0.15 \%$ Bacto Gelatin [Difco]). Another test involved the use of Columbia agar made up from its separate components but with starch omitted (see below). Tests for protease activity on fibrin from other mammal species (humans, horses, and dogs) were also carried out. Fibrin plates prepared with fibrinogens from human plasma (catalog no. F-4129; fraction I, type III; Sigma), from horse plasma (catalog no. F-9516; fraction I; Sigma), and from dog plasma (catalog no. F-7128; Sigma) as described above were used, but plasma was not added.

\section{RESULTS}

PAGE of whole-cell proteins. A total of 129 streptococci were examined. Duplicate protein extracts of several strains were prepared to check the reproducibility of the growth conditions and the preparation of the extracts. The level of correlation between duplicate protein patterns was at least $94 \%$ (data not shown). After visual comparison of the protein patterns with the dendrogram obtained after numerical comparison and clustering of the profiles, we identified 12 stable clusters above a similarity level of $84 \%$ (Fig. 1). Cluster I comprised $45 S$. dysgalactiae strains, including type strain LMG 16023 , which clustered above a similarity level of $84.8 \%$. These strains were isolated from various animals and belong to serogroups C and L (Fig. 1; Table 1). Cluster III comprised $35 S$. dysgalactiae strains that clustered above a similarity level of $84.7 \%$. All of the cluster III strains were isolated from human sources and belong to serogroups $\mathrm{C}$ and $\mathrm{G}$ (Fig. 1; Table 1). As shown in Fig. 1, the reference strains of all of the other streptococcal species examined constituted homogeneous clusters, as follows: $S$. canis (cluster II; grouping above a similarity level of $87.2 \%$ ), Streptococcus pyogenes (cluster IV; grouping above a similarity level of $88.9 \%$ ), Streptococcus uberis (cluster V; grouping above a similarity level of $84.1 \%$ ), S. agalactiae (cluster VI; grouping above a similarity level of $85.8 \%$ ), Streptococcus hyointestinalis (cluster VII; grouping above a similarity level of $91.7 \%$ ), Streptococcus parauberis (cluster VIII; grouping above a similarity level of $96.3 \%$ ), Streptococcus porcinus (cluster IX; grouping above a similarity level of $88.7 \%$ ), Streptococcus anginosus (cluster X; grouping above a similarity level of $89.2 \%$ ), and S. equi (cluster XI; grouping above a similarity level of $88.4 \%$ ). Cluster XII comprised the two Streptococcus iniae reference strains and the type strain of Streptococcus shiloi, which grouped above a correlation level of $93.2 \%$. Figure 2 shows the whole-cell protein patterns of representative strains belonging to different clusters.

Physiological tests. Physiological tests were performed on all $80 \mathrm{~S}$. dysgalactiae strains. The serotypes of these strains according to the Lancefield scheme are shown in Table 1. All of the strains except the bovine group $\mathrm{C}$ strains which were isolated from tonsils (strains LMG 14602, LMG 14604, and LMG 14605) and milk (strains LMG 15748, LMG 15749, LMG 16024, LMG 16025, and LMG $16023^{\mathrm{T}}$ [T = type strain]) and a single group $\mathrm{C}$ strain isolated from a pig (strain LMG 15744) exhibited beta-hemolysis on bovine blood agar. The bovine group $\mathrm{C}$ strains were alpha-hemolytic or nonhemolytic. Porcine strain LMG 15744 was also alpha-hemolytic.

All 35 strains belonging to cluster III as determined by the whole-cell protein analysis (Fig. 1) produced streptokinase activity on human plasminogen. This was shown by the presence of neat and easily visible zones of clearing surrounding streptococcal growth spots on bovine fibrin-plasma plates. Most reaction zones were visible after 1 day of incubation, but in a few cases, when growth was weak, they appeared only after 2 days. No strain reacted with plasminogens from the five animal species tested. The strains also did not cause fibrinolysis on bovine fibrin plates without plasma, and they were all negative in protease tests on milk agar and gelatin agar. The amylase reaction, which is visible with the naked eye on Columbia agar base without fibrin, did not interfere with the streptokinase tests because the starch contained in the medium was hydrolyzed and could not be demonstrated, even with iodine, following the addition of the fibrin preparations to the Columbia agar base. Tests performed with laboratory-prepared Columbia agar without starch gave the same results.

One equine protein electrophoretic cluster I strain (LMG 15824) produced some clearing on bovine fibrin-horse plasma plates. This clearing was visible beneath the growth spot only when the spot was washed off after 2 days of incubation. None of the 44 other cluster I strains tested, including the 4 other equine Lancefield group $\mathrm{C}$ strains, gave evidence of streptokinase activity in the plate tests. All of these strains except the eight bovine group $\mathrm{C}$ strains listed above were negative in tests performed on bovine fibrin plates with or without different types of plasma, including human plasma, and on milk agar and gelatin agar. These strains lacked streptokinase activity. However, the eight alpha- or nonhemolytic bovine group $\mathrm{C}$ strains (including the $S$. dysgalactiae type strain) produced ex- 


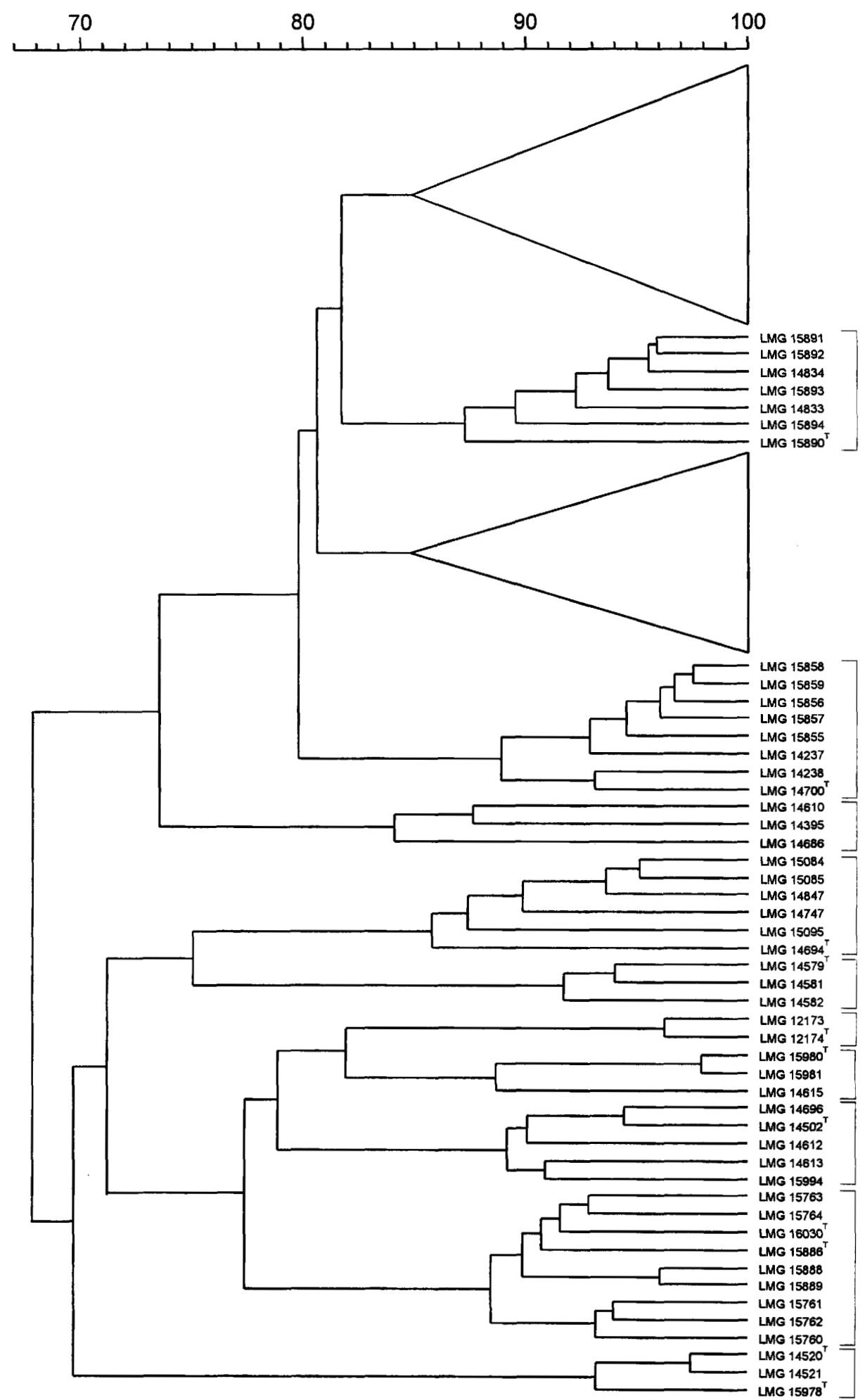

\section{S. dysgalactiae subsp. dysgalactiae (45 strains)}

II S. canis

\section{S. dysgalactiae} subsp. equisimilis (35 strains)

IV S. pyogenes

\section{S. uberis}

VI S. agalactiae

VII S. hyointestinalis

VIII S. parauberis

IX S. porcinus

$X S$. anginosus

XI S. equi

FIG. 1. Dendrogram derived from unweighted pair group average linkage of correlation coefficients (expressed for convenience as percentages) for the whole-cell protein patterns of all of the strains examined. Roman numerals are cluster numbers (see text).

actly the same fibrinolysis zones on bovine fibrin plates with and without human or animal plasma. This indicates that the lysis observed was not due to plasmin activity resulting from the action of streptokinase on plasminogen, but was caused by proteolytic enzymes. Indeed, the same strains lysed casein on milk agar plates; however, they were not active on gelatin.

All of the cluster III strains produced neat zones of fibrinolysis on human fibrin-containing plates without added plasma, 


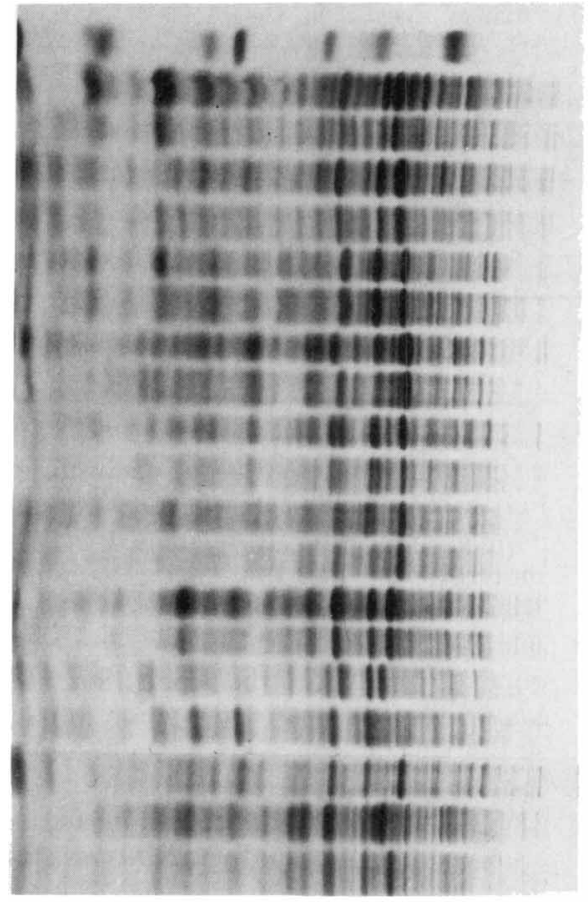

A
S. dysgalactiae subsp. dysgalactiae LMG $16023 \mathrm{~T}$
S. dysgalactiae subsp. dysgalactiae LMG 15751
S. dysgalactiae subsp. equisimilis LMG $16026 \mathrm{~T}$
S. dysgalactiae subsp. equisimilis LMG 15840
S. equi subsp. equi LMG $15886 \mathrm{~T}$
S. equi subsp. zooepidemicus LMG 16030T
S. agalactiae LMG $14694 \mathrm{~T}$
S. agalactiae LMG $15(184$
S. anginosus LMG 15994
S. anginosus LMG 14612
S. porcinus LMG 14615
S. porcinus LMG $1598(1 \mathrm{~T}$
S. iniae LMG $1452(1 \mathrm{~T}$
S. shiloi LMG $15978 \mathrm{~T}$
S. pyogenes LMG 147()$(1 \mathrm{~T}$
S. canis LMG $1589(1 \mathrm{~T}$
S. hyointestinalis LMG $14579 \mathrm{~T}$
S. uberis LMG $14686 \mathrm{~T}$
S. parauberis LMG $12174 \mathrm{~T}$

FIG. 2. Protein profiles of representative strains of each electrophoretic cluster. For $S$. agalactiae, S. anginosus, and $S$. porcinus, protein patterns of both human and animal isolates are shown (Table 1). The molecular weight markers used (lane A) were (from left to right) lysozyme (molecular weight, 14,500), trypsin inhibitor $(20,100)$, trypsinogen $(24,000)$, carbonic anhydrase $(29,000)$, glyceraldehyde-3-phosphate dehydrogenase $(36,000)$, egg albumin $(45,000)$, and bovine albumin $(66,000)$.

while none of the cluster I strains exhibited any visible activity on this substrate. On canine fibrin plates, seven of the eight bovine cluster I strains produced wide zones of lysis which were identical to the zones that they produced on bovine fibrin agar plates and on milk agar plates. The remaining strains were not active on this type of fibrin. All of the strains studied were negative on horse fibrin.

\section{DISCUSSION}

Selection of $S$. dysgalactiae strains. $S$. dysgalactiae consists of at least five distinct subgroups on the basis of serogroups, biotypes, and hosts $(5,7)$. In the present study, the sources of the strains used included humans, horses, cows (and milk), pigs, dogs, chickens, and an iguana (Table 1). These isolates represented nonhemolytic, alpha-hemolytic and beta-hemolytic $S$. dysgalactiae strains and serogroups C, G, and L. Thus, the strains used (Table 1) reflect the known diversity within this species.

Differentiation of streptococci by whole-cell protein electrophoresis. $S$. agalactiae, $S$. canis, $S$. dysgalactiae, $S$. equi, $S$. hyointestinalis, $S$. iniae, $S$. parauberis, $S$. porcinus, $S$. pyogenes, and $S$. uberis constitute the pyogenic species group as determined by $16 \mathrm{~S}$ rRNA sequence analysis (1). Other streptococcal species are more remotely related to members of this group. In the present study, we included representative strains of all of these taxa as reference strains. In addition, $S$. anginosus was also included, as this species also comprises Lancefield group $\mathrm{C}$ and $\mathrm{G}$ strains. $S$. dysgalactiae strains were easily differentiated from other streptococci by whole-cell protein electrophoresis (Fig. 1 and 2). However, these organisms fell into two major clusters, with all of the animal isolates constituting one cluster (cluster I) (Fig. 1) and the human isolates constituting the other (cluster III) (Fig. 1). Clusters I and III contain five (LMG $16023^{\mathrm{T}}$ through LMG 16025, LMG 16027, and LMG 16029) and one $\left(\right.$ LMG $16026^{\mathrm{T}}$ ) reference strains, respectively, which were included in extensive DNA-DNA hybridization studies (9) (Fig. 1; Table 1). The DNA-DNA binding values obtained by using the membrane filter technique between these strains and other $S$. dysgalactiae reference strains ranged from 66 to $100 \%$ (9), indicating that they form a homogeneous species. As determined by protein electrophoresis, these strains are representative of their respective clusters, and we therefore concluded that the two clusters indeed represent a genotypically coherent species despite salient protein electrophoretic differences. These differences occur mainly in the 45,000 - to 55,000 -molecularweight region (molecular weights were estimated roughly after comparison with the molecular weight ladder [Fig. 2]). These two clusters apparently represent different electrophoretic types of $S$. dysgalactiae, and the origins of the $S$. dysgalactiae strains apparently are useful for identifying the electrophoretic subgroups. The collection of 80 strains examined in this study was selected in such a way that it represented the biochemical and serological diversity within the species, and we assume that it is therefore representative of the entire species. From an epidemiological point of view, these findings indicate that humans are never or only rarely infected by $S$. dysgalactiae strains from animals. Interestingly, the animals from which strains have been isolated are not only farm animals, but also companion animals. Consequently, not only farm workers but also pet owners are regularly exposed to $S$. dysgalactiae strains of animal origin, yet are not clinically infected. The improved method for distinguishing between animal-associated and humanassociated $S$. dysgalactiae strains described here should help trace possible zoonotic infections.

The reference strains of $S$. agalactiae, $S$. anginosus, $S$. canis, $S$. equi, $S$. iniae, $S$. hyointestinalis, $S$. parauberis, $S$. porcinus, $S$. pyogenes, and $S$. uberis constitute separate clusters on the dendrogram (Fig. 1). The $S$. shiloi type strain forms a single cluster 
TABLE 2. Characteristics that differentiate $S$. dysgalactiae subspecies

\begin{tabular}{|c|c|c|c|c|c|c|c|c|}
\hline \multirow{2}{*}{ Taxon } & \multirow{2}{*}{ Hemolysis } & \multirow{2}{*}{$\begin{array}{l}\text { Lancefield } \\
\text { carbohydrate } \\
\text { antigens }\end{array}$} & \multirow{2}{*}{$\begin{array}{l}\text { Strepokinase } \\
\text { activity on } \\
\text { human plas- } \\
\text { minogen }\end{array}$} & \multirow{2}{*}{$\begin{array}{l}\text { Proteolysis } \\
\text { of human } \\
\text { fibrin }\end{array}$} & \multicolumn{2}{|c|}{$\begin{array}{l}\text { Acid production } \\
\text { from }{ }^{a} \text { : }\end{array}$} & \multirow{2}{*}{$\begin{array}{l}\alpha-L-G l u t a m a t e \\
\text { aminopeptidase } \\
\text { activity }^{a}\end{array}$} & \multirow{2}{*}{$\begin{array}{l}\text { L-Prolyl-L-argi- } \\
\text { nine aminopepti- } \\
\text { dase activity }{ }^{a}\end{array}$} \\
\hline & & & & & Sorbitol & Glycogen & & \\
\hline S. dysgalactiae subsp. dysgalactiae & $\begin{array}{l}\text { Alpha, beta, or } \\
\text { none }\end{array}$ & $\mathrm{C}, \mathrm{L}$ & $-{ }^{b}$ & - & $\mathrm{D}$ & $\mathrm{D}$ & $\mathrm{D}$ & + \\
\hline S. dysgalactiae subsp. equisimilis & Beta & $\mathrm{C}, \mathrm{G}$ & + & + & - & - & + & D \\
\hline
\end{tabular}

${ }^{a}$ Data from reference 7

${ }^{b}-$, negative; + , positive; D, strain dependent.

with the $S$. iniae strains at a correlation level of $93.2 \%$, confirming the reported synonymy of these two species (8). As illustrated in Fig. 1 and 2, S. equi subsp. equi and $S$. equi subsp. zooepidemicus cannot be differentiated by whole-cell protein electrophoresis. In contrast to the $S$. dysgalactiae strains, human and animal isolates of $S$. agalactiae, $S$. anginosus, and $S$. porcinus do not seem to exhibit significant differences in their whole-cell protein patterns (Table 1; Fig. 2).

Phenotypic differentiation of $S$. dysgalactiae strains. Despite the superficial resemblance of the assay methods, direct proteolytic activity on human fibrin and streptokinase activity on human plasminogen are two different characteristics which can be used as easy ways to distinguish between human and animal isolates of $S$. dysgalactiae in diagnostic laboratories. McCoy and coworkers (13) found that streptokinases of different species obtained from different hosts were antigenically related. In their study, all of the group $\mathrm{C}$ strains tested, including strains referred to as $S$. equisimilis, $S$. equi subsp. equi, and $S$. equi subsp. zooepidemicus, had surface receptors that bound human, equine, and porcine plasmins. Human $S$. equisimilis strains were active only on human plasminogen, and this finding was confirmed and extended in the present study with avian, bovine, and canine plasmas. However, equine $S$. equisimilis strains and strains from the two $S$. equi subspecies activated horse plasma, while porcine $S$. equisimilis strains were active on porcine plasminogen and, very weakly, also on human plasminogen. In the study of McCoy et al. (13), plasminogen activator activity was identified by hydrolysis of a plasmin-specific chromogenic substrate. This was detected in growth patches by a solid-phase assay. In our study, the enzyme was detected by lysis of fibrin, the normal substrate of plasmin. The difference in test results may be due to differences in the sensitivities of the two test systems.

Taxonomic status of $S$. dysgalactiae strains of human origin and animal origin. Species level identification of $S$. dysgalactiae strains is complex and provides little information for clinicians or epidemiologists. Beta-hemolytic group L $S$. dysgalactiae strains and alpha-hemolytic group $\underset{C}{C} S$. dysgalactiae strains colonize or infect only animals, whereas beta-hemolytic group $\mathrm{G} S$. dysgalactiae strains colonize or infect only humans. Betahemolytic group C $S$. dysgalactiae strains occur in humans and animals, but the animal isolates do not occur in humans and vice versa. Clearly, from a practical point of view, $S$. dysgalactiae consists of two distinct subpopulations of strains, which produce very different whole-cell protein electrophoretic patterns, as demonstrated in Fig. 1. The protein electrophoretic differences are comparable to the differences between distinct species in the group containing the pyogenic streptococci (Fig. 1 and 2). In contrast, human and animal isolates of several other members of this species group do not differ significantly (Fig. 1 and 2; Table 1). The two subpopulations of $S$. dysgalactiae can also be readily identified by phenotypic tests, as described above. In our opinion, these observations warrant distinct taxonomic status for each subpopulation of strains. As Farrow and Collins (9) demonstrated that both of the subpopulations belong to a single species, we propose that they should be classified as distinct subspecies of $S$. dysgalactiae. The name $S$. dysgalactiae subsp. dysgalactiae is used for the subpopulation of strains of animal origin because this subpopulation includes the type strain. Historically, the name $S$. equisimilis was given to human isolates resembling $S$. equi strains which occurred in animals, and therefore it is logical to use the name $S$. dysgalactiae subsp. equisimilis for the $S$. dysgalactiae strains of human origin. In addition, this avoids creating more confusion by the introduction of a new name.

$S$. dysgalactiae subsp. dysgalactiae strains can be distinguished from $S$. dysgalactiae subsp. equisimilis strains by proteolysis of human fibrin, by the results of the human plasminogen-streptokinase test, by origin, and by whole-organism protein electrophoretic patterns. Lancefield serogrouping is also of value for differentiating the two subspecies; group L streptococci always belong to $S$. dysgalactiae subsp. dysgalactiae, while group $\mathrm{G}$ streptococci of human origin belong to $S$. dysgalactiae subsp. equisimilis (all animal group $\mathrm{G}$ strains described to date belong to $S$. canis). These identifications can be confirmed by physiological tests $(6,7)$. Also, for group C streptococci, origin can be used for presumptive identification; however, the strains should first be differentiated from $S$. equi and "S. milleri" $(7,9)$. Finally, Efstratiou et al. (7) described additional phenotypic tests that can be used to differentiate the two subspecies (Table 2).

Description of $S$. dysgalactiae subsp. dysgalactiae subsp. nov. The description of $S$. dysgalactiae subsp. dysgalactiae is the same as the description given by Farrow and Collins (9) for $S$. dysgalactiae, with following exceptions. Most strains are beta-hemolytic, but alpha-hemolytic and nonhemolytic strains occur, especially in bovine sources. Strains may react with Lancefield group C or L antigen but not with Lancefield group $\mathrm{G}$ antigen. Streptokinase activity on human plasminogen and proteolytic activity on human fibrin do not occur. The habitat is the respiratory and genital tracts of various animals, but apparently not humans.

Description of $S$. dysgalactiae subsp. equisimilis subsp. nov. The description of $S$. dysgalactiae subsp. equisimilis is the same as the description given by Farrow and Collins (9) for $S$. dysgalactiae, with following exceptions. Strains are beta-hemolytic and may react with Lancefield group $\mathrm{C}$ or $\mathrm{G}$ antigen but not with Lancefield group $\mathrm{L}$ antigen. Streptokinase activity occurs on human plasminogen, and proteolytic activity occurs on human fibrin. The habitat is the respiratory tracts and vaginas of humans. The type strain is LMG 16026 (= NCFB 1356), which belongs to Lancefield group G.

\section{ACKNOWLEDGMENTS}

We thank Urbain Torck and Dirk Dewettinck for excellent technical assistance and all depositors of strains listed in Table 1. 
P.V. is indebted to the National Fund for Scientific Research (Belgium) for a position as a postdoctoral research fellow. K.K. is indebted to the Fund for Medical Scientific Research, Belgium, for research and personnel grants. Part of this work was supported by the Federal Office for Scientific, Technical and Cultural Affairs of the Belgian State.

\section{REFERENCES}

1. Bentley, R. W., J. A. Leigh, and M. D. Collins. 1991. Intrageneric structure of Streptococcus based on comparative analysis of small-subunit rRNA sequences. Int. J. Syst. Bacteriol. 41:487-494.

2. Breed, R. S., E. G. D. Murray, and A. P. Hitchens. 1948. Manual of determinative bacteriology, 6th ed., p. 318-319. Williams and Wilkins, Baltimore.

3. Carmeli, Y., and K. L. Ruoff. 1995. Report of cases of and taxonomic considerations for large-colony-forming Lancefield group C streptococcal bacteremia. J. Clin. Microbiol. 33:2114-2117.

4. Costas, M. 1992. Classification, identification, and typing of bacteria by the analysis of their one-dimensional polyacrylamide gel electrophoretic protein patterns, p. 351-408. In A. Chambrach, M. J. Dunn, and B. J. Radola (ed.) Advances in electrophoresis, vol. 5. VCH Verlagsgesellschaft, Weinheim, Germany.

5. Devriese, L. A. 1991. Streptococcal ecovars associated with different animal species: epidemiological significance of serogroups and biotypes. J. Appl. Bacteriol. 71:478-483.

6. Devriese, L. A., J. Hommez, R. Kilpper-Bälz, and K.H. Schleifer. 1986. Streptococcus canis sp. nov.: a species of group $\mathrm{G}$ streptococci from animals. Int. J. Syst. Bacteriol. 36:422-425.
7. Efstratiou, A., G. Colman, G. Hahn, J. F. Timoney, J. M. Boeufgras, and D. Monget. 1994. Biochemical differences among human and animal streptococci of Lancefield group C or group G. J. Med. Microbiol. 41:145-148.

8. Eldar, A., P. F. Frelier, L. Assenta, P. W. Varner, S. Lawhon, and H. Bercovier. 1995. Streptococcus shiloi, the name for an agent causing septicemic infection in fish, is a junior synonym of Streptococcus iniae. Int. J. Syst. Bacteriol. 45:840-842.

9. Farrow, J. A. E., and M. D. Collins. 1984. Taxonomic studies on streptococci of serological groups C, G and L and possibly related taxa. Syst. Appl. Microbiol. 5:483-493.

10. Frost, W. D., and M. A. Engelbrecht. 1940. The streptococci. Willdorf Book Co., Madison, Wis.

11. Garvie, E. I., J. A. E. Farrow, and M. D. Collins. 1983. Streptococcus dysgalactiae (Diernhofer) nom. rev. Int. J. Syst. Bacteriol. 33:404 405.

12. Lancefield, R. C. 1933. A serological differentiation of human and other groups of hemolytic streptococci. J. Exp. Med. 59:571-591.

13. McCoy, H. E., C. C. Broder, and R. Lottenberg. 1991. Streptokinases produced by pathogenic group C streptococci demonstrate species-specific plasminogen activation. J. Infect. Dis. 164:515-521.

14. Pot, B., P. Vandamme, and K. Kersters. 1994. Analysis of electrophoretic whole-organism protein fingerprints, p. 493-521. In M. Goodfellow and A. G. O'Donnell (ed.), Modern microbial methods. Chemical methods in prokaryotic systematics. J. Wiley and Sons, Ltd., Chichester, United Kingdom.

15. Skerman, V. B. D., V. McGowan, and P. H. A. Sneath (ed.). 1980. Approved lists of bacterial names. Int. J. Syst. Bacteriol. 30:225-420. 